Supplementary Information for:

\title{
Exploring the Catalytic Mechanism of Human Glutamine Synthetase by Computer Simulations
}

Federico M. Issoglio, ${ }^{a}$ Nicolas Campolo,,${ }^{b, c}$ Ari Zeida, ${ }^{a}$ TilmanGrune, ${ }^{e}$ Rafael Radi,,${ }^{b, c}$ Dario A. Estrin, ${ }^{a^{*}}$ Silvina Bartesaghi, ${ }^{b, c, d^{*}}$

åDepartamento de QuímicaInorgánica, Analítica y Química-Física and INQUIMAE-CONICET, Facultad de Ciencias Exactas y Naturales, Universidad de Buenos Aires, Buenos Aires, Argentina.

${ }^{\mathrm{b}}$ Departamento de Bioquímica and ${ }^{\mathrm{c}}$ Center for Free Radical and Biomedical Research, Facultad de Medicina, Universidad de la República, Montevideo, Uruguay.

${ }^{\mathrm{d}}$ Departamento de EducaciónMédica,Facultad de Medicina, Universidad de la República, Montevideo, Uruguay.

${ }^{\mathrm{e}}$ German Institute of Human Nutrition (DIfE) Potsdam-Rehbrücke, Department of Molecular Toxicology, Arthur-Scheunert-Allee 114-116, 14558 Nuthetal, Germany.

* To whom correspondence should be addressed. e-mail: sbartesa@fmed.edu.uy, Telephone: (5982)9249561, Fax: (5982)9249563; e-mail: dario@qi.fcen.uba.ar, Telephone:

(5411)45763358, Fax: (5411)45763341. 

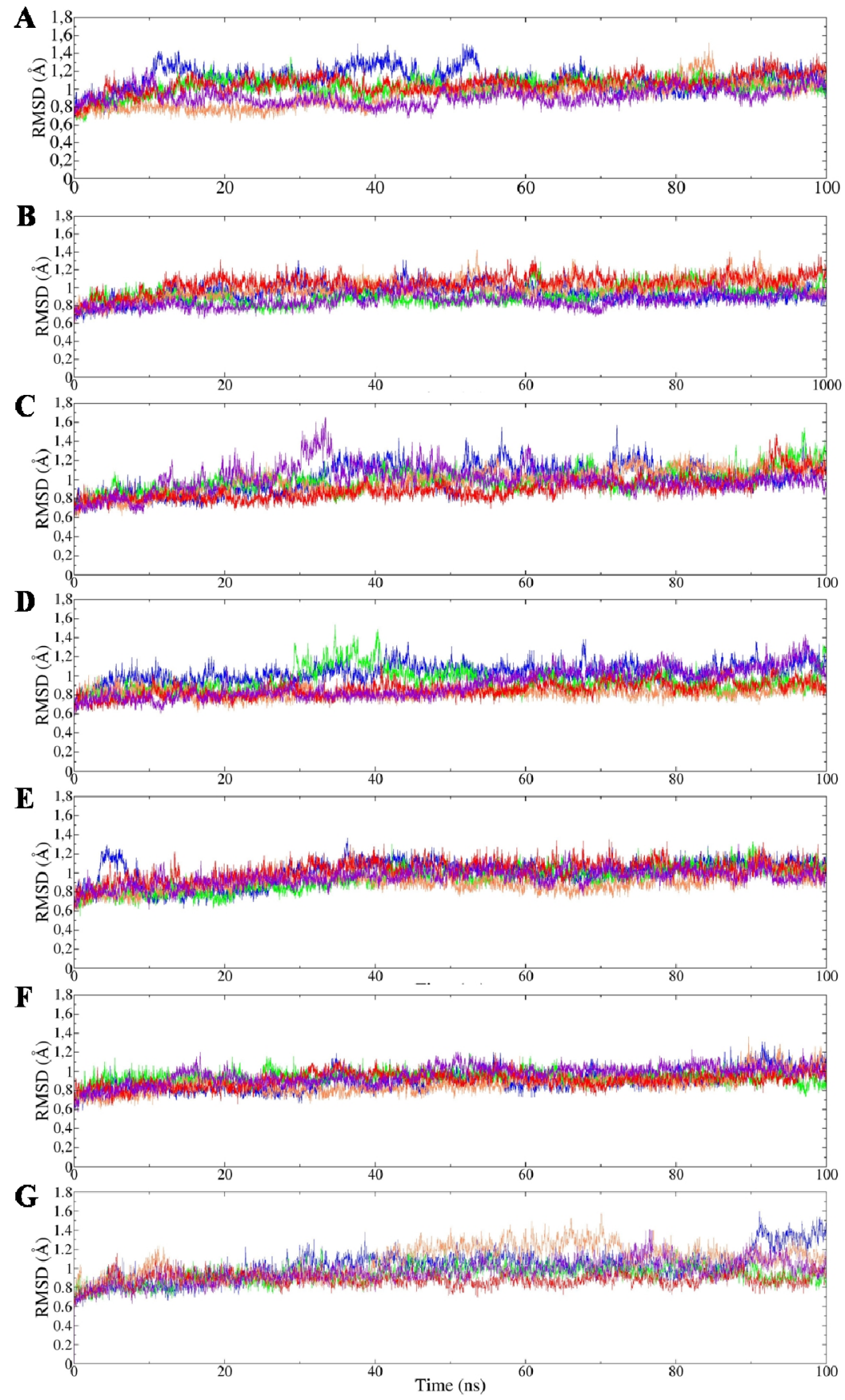

Figure S1. RMSD calculations for $H_{s} \mathrm{GS}$ backbone atoms relative to the optimized structures, colored by subunit of the pentamer. In panels $\mathbf{A}$ to $\mathbf{G}$ the RMSD for simulations 1 to 7 are represented. In all the systems the RMSD through $100 \mathrm{~ns}$ simulations is hardly above $1.2 \AA$. 

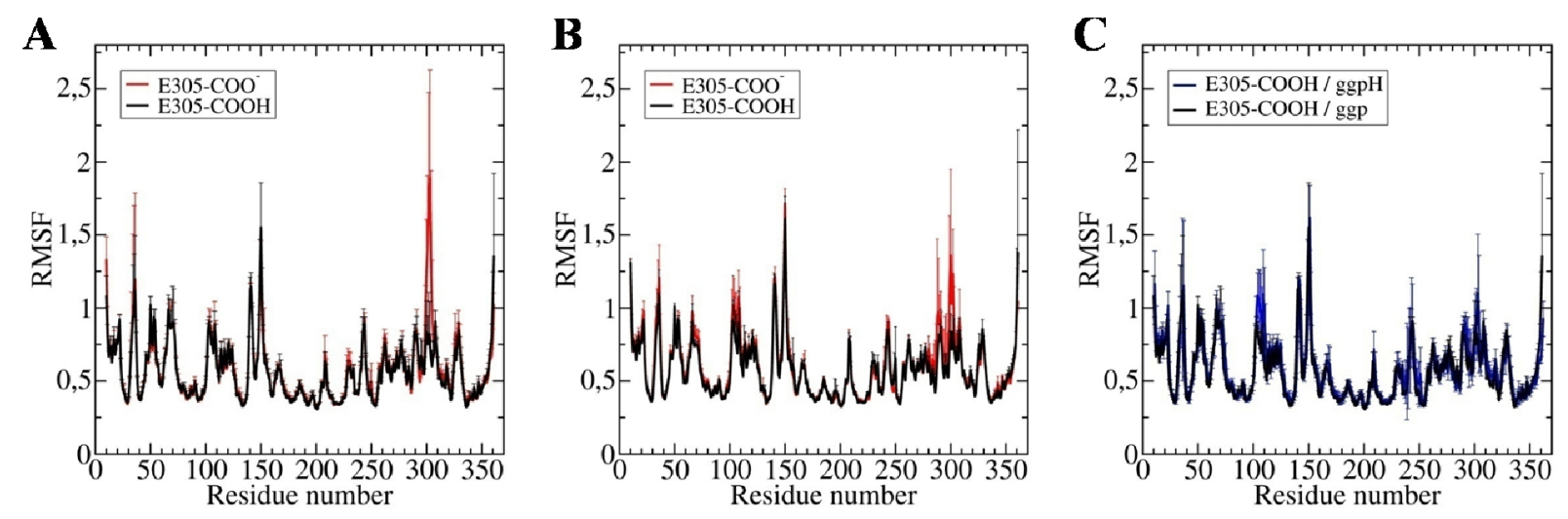

Figure S2. A) Per residue backbone atoms RMSF for 100 ns simulations 3 and 4, where $H s G S$ is complexed with ADP, $\mathrm{Mn}^{2+}, \gamma$-Glu-P and $\mathrm{NH}_{3}$, and E305 is protonated (black line) or deprotonated (red line). Same analysis was made for simulations 5 and 6 , in where $\mathrm{NH}_{4}$ is the nucleophile (B), and between simulations 4 and 7, where in the last $\gamma$-Glu-P-H is the intermediate and $\mathrm{NH}_{3}$ the nucleophile $(\mathbf{C})$. Every residue RMSF represents the mean of the five values corresponding to each subunit, and the error bars are the standard deviation. 

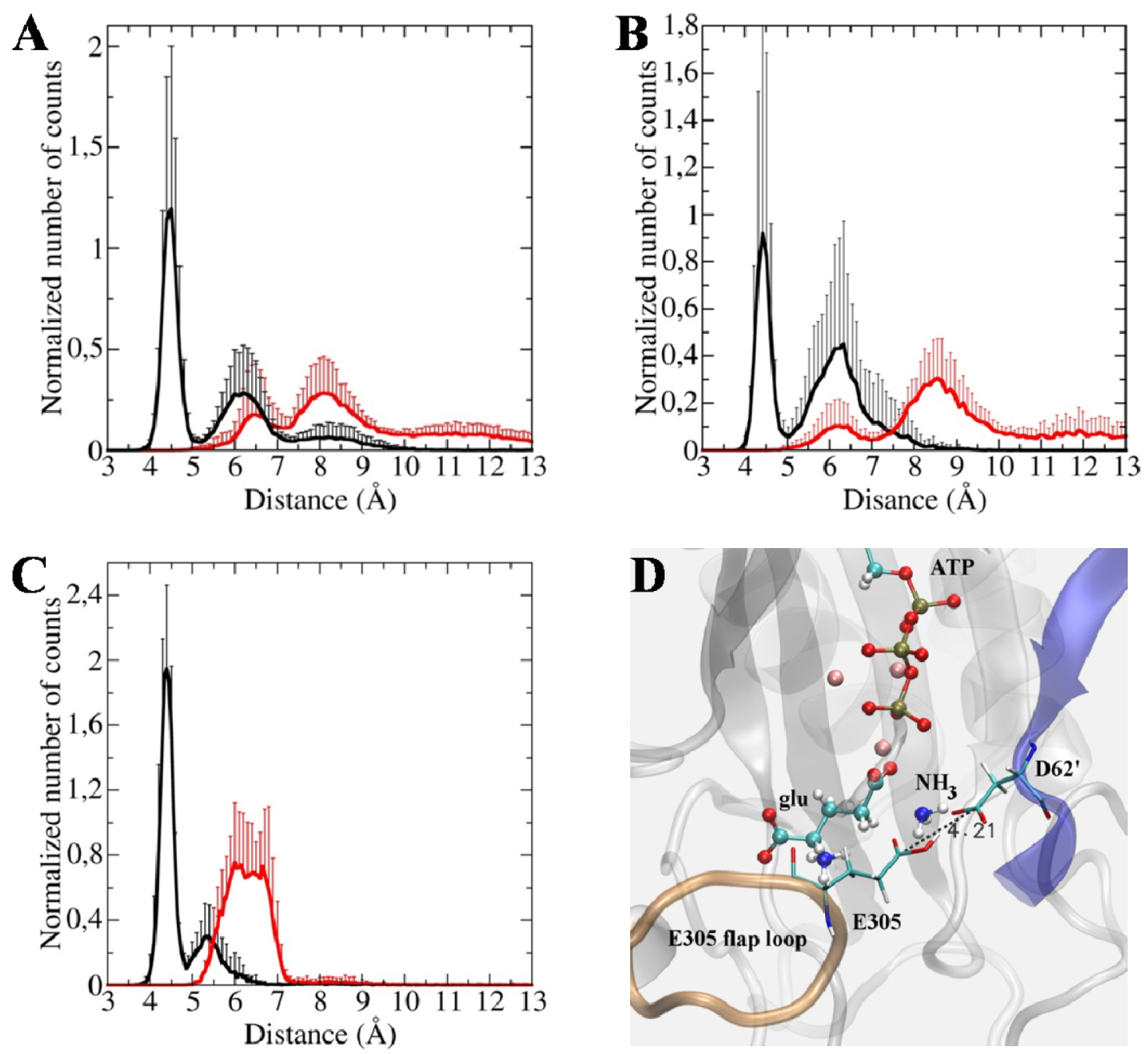

Figure S3. Histograms for distance distribution between $\delta$-carbon of E305 and $\gamma$-carbon of D62' used to visualize structural stability of the E305 flap loop, where simulations 1 with 2 (A), 3 with 4 (B) and 5 with 6 (C) are compared. Black and red lines corresponds to $100 \mathrm{~ns}$ simulations average data collected from the five subunits, in which E305 is protonated or deprotonated respectively. It is important to point out that the interaction is considered broken for distances higher than $5 \AA$. D). Graphical representation of the active site with ATP glu and $\mathrm{NH}_{3}$. For clarity, mainly one subunit is represented colored with grey, and with E305 flap loop colored in transparent orange. Only a segment of the other subunit involved in the active site is shown, wich contains D62' (in transparent blue). 

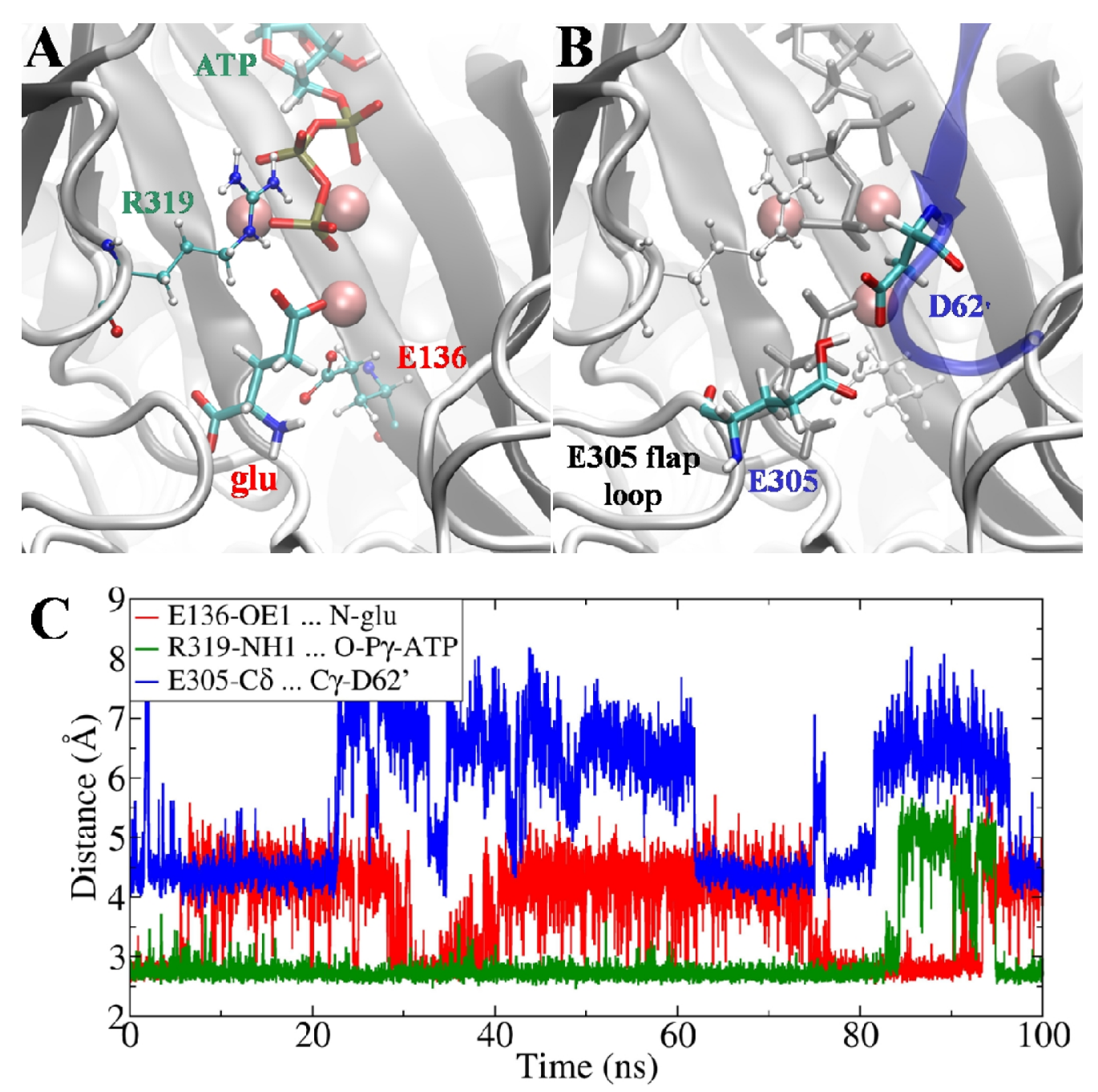

Figure S4. A) Graphical representation of $H_{s} \mathrm{GS}$ active site. ATP and glutamate are shown with "licorice" representation, and residues R319 and E136 are depicted with "ball and stick" representation. B) Position of E305 and D62' in the closed conformation of E305 flap loop. A segment of the $\beta$-sheet to with D62' belongs is depicted in transparent blue. C) Analysis over 100 ns simulation of distances between E136 carboxylate and amine group from glutamate (red line), between nitrogen atom of the lateral chain of R319 and oxygen form $\gamma$-phosphate (green line), and between delta and gamma carbons from the side chains of E305 and D62' (blue line). The analysis shown corresponds to one of the five pentamer active sites. 


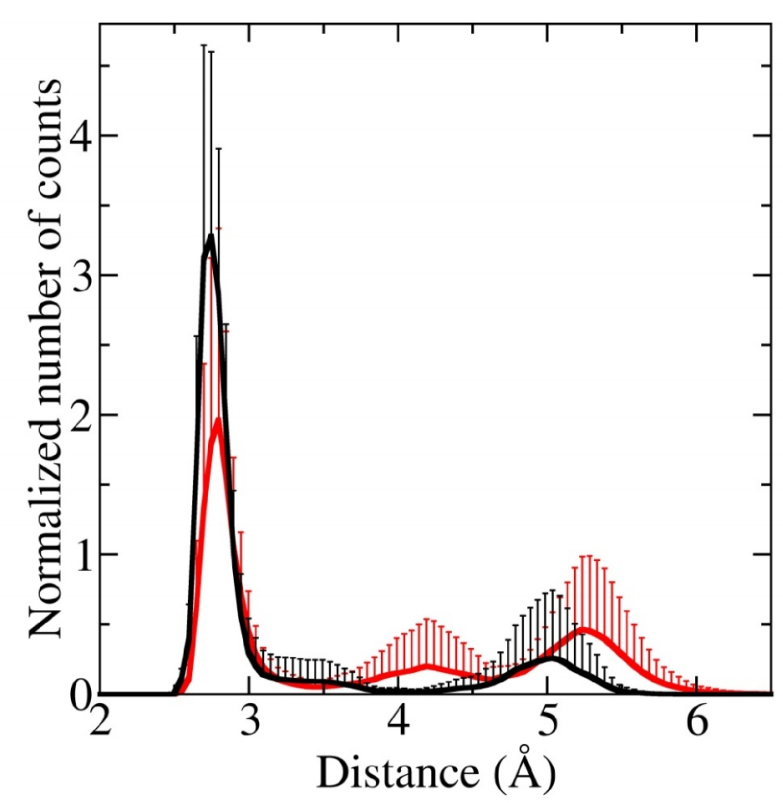

Figure S5. Histograms for distance distribution between $\gamma$-phosphate and R319 guanidinium group. Black and red lines corresponds to 100 ns simulations average data collected from the five subunits, in which E305 is protonated or deprotonated respectively. Note that the lack of hydrogen bond between E305 and D62' diminishes the population of conformations in which R319 is directly interacting with $\gamma$-phosphate from ATP (i.e. distance below $3 \AA$ ). Error bars represent the standard deviation. 


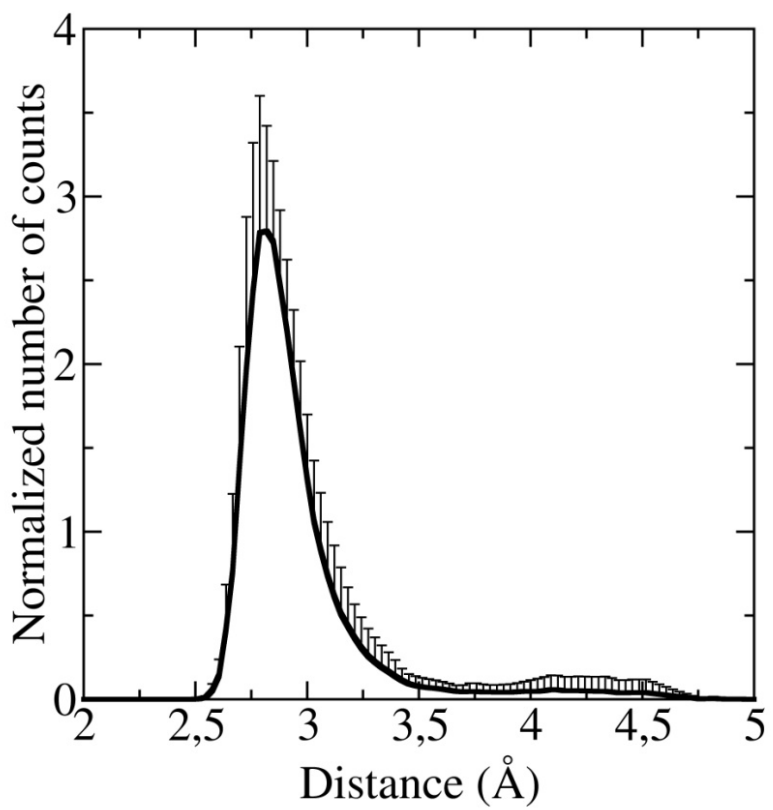

Figure S6. Analysis of distance between $\mathrm{NH}_{4}{ }^{+}$nitrogen atom and an oxygen atom from $\gamma$-Glu-P phosphate moiety in simulation 6 ( $\mathrm{HsGS}+\mathrm{ADP}+\gamma$-Glu-P $+\mathrm{NH}_{4}{ }^{+}$, and with E305 protonated). A strong hydrogen bond is present through $100 \mathrm{~ns}$ simulation and among all subunits. Error bars represents standard deviation of the average value (solid line) computed with data obtained from the five active sites of the pentamer. 


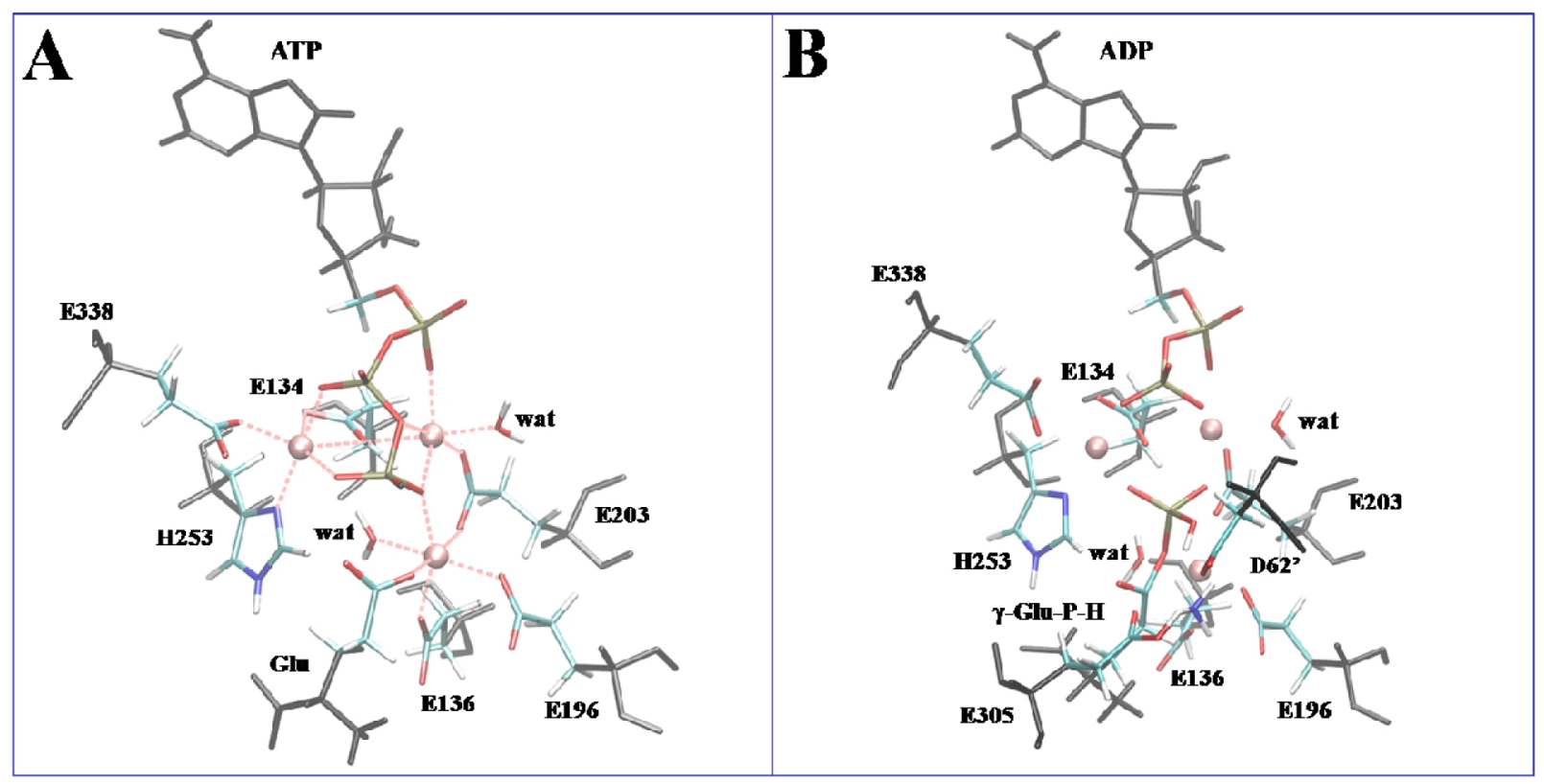

Figure S7. Graphical description of the QM/MM boundaries for simulation 2 (A) and simulation 7 (B). Atoms included in the QM system are colored by atom type, and the rest is colored in black. $\mathrm{Mn}^{2+}$ ions are depicted as pink spheres. In panel $\mathrm{A}$ the coordination of $\mathrm{Mn}^{2+}$ ions is represented with pink dashed lines. In both situations QM system comprises residues E134, E136, E196, E203, H253 and E338 lateral chains excluding backbone atoms and $\alpha$-carbons. For ATP and ADP molecules the nitrogenous base and the ribose to the C4' carbon atom were not included in the QM system. When the second step of the reaction was studied (B), ammonia molecule was included in the QM system, as well as residues E305 and D62' (using the same criteria mentioned above for the QM/MM boundary).

3D Animation Specifications (Issoglio_etat_1.avi and Issoglio_etal_2.avi). The animations were prepared with representative trajectories from MSMD simulations, corresponding to the first step of the reaction ( $\gamma$-phosphate transfer from ATP to Glu), and for the second step of the reaction (nucleophilic attack of $\mathrm{NH}_{3}$ to the $\delta$-carbon of the reaction intermediate $\gamma$-Glu-P-H). 